\title{
Optimizing magneto-optical effects in the ferromagnetic semiconductor GaMnAs
}

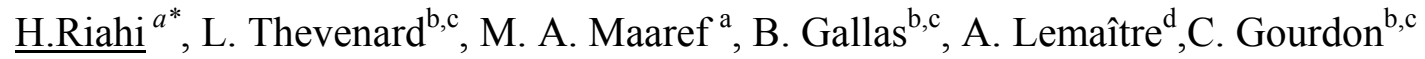 \\ ${ }^{a}$ Laboratoire Matériaux Molécules et Applications, IPEST, Université de Carthage, La \\ Marsa, Tunisie \\ ${ }^{b}$ CNRS, UMR7588, Institut des Nanosciences de Paris, 4 place Jussieu, 75005 Paris, \\ France \\ 'Sorbonne Universités, UPMC Université Paris 06, UMR7588, 4 place Jussieu, 75005 \\ Paris, France. \\ ${ }^{d}$ Laboratoire de Photonique et de Nanostructures - CNRS, Route de Nozay, 91460 \\ Marcoussis, France.
}

e-mail: hassenriahi1987@gmail.com

Keywords : magnetic semiconductors, magneto-optical Kerr effect

\begin{abstract}
:
A trilayer of the ferromagnetic semiconductor GaMnAs, a $\mathrm{SiO}_{2}$ buffer layer and a piezoelectric $\mathrm{ZnO}$ layer, is investigated in view of its use in device implementation to study surface acoustic wave-assisted magnetization switching. The magneto-optical properties: Kerr rotation and ellipticity and magnetic contrast in Kerr microscopy images are investigated as a function of temperature. While the $\mathrm{ZnO}$ layer prevents any good quality imaging of magnetic domains, we show that with the $\mathrm{SiO}_{2}$ layer only the polar Kerr rotation and the magnetic contrast are increased by a factor of 2 . This result is in good quantitative agreement with calculations using an optical interference model and could be further improved. The detrimental effects of the dielectric layers capping on the Curie temperature and coercive field of the GaMnAs layer can be kept to a reasonable level.
\end{abstract}

\section{Introduction :}

The ferromagnetic semi-conductor $(\mathrm{Ga}, \mathrm{Mn}) \mathrm{As}$ has received considerable attention in the recent years, as a promising material for future semiconductor spintronic devices [1,2]. It has become a test-bed material for magnetization manipulation by static strain $[3,4]$, electric field [5], light pulses, [6, 7, 8, 9], acoustic (strain) pulses [10, 11], and very recently by surface acoustic waves (SAW) $[\mathbf{1 2 , 1 3 ]}$. This has been made possible thanks to the large sensitivity of the magnetic anisotropy to strain $[14,15]$. Strain waves like SAWs have been identified as a powerful tool for magnetization switching in magneto-strictive materials [16, 17, 18]. In GaMnAs, it was recently demonstrated that magnetization precession can be resonantly driven by a SAW when both the magnon and phonon frequencies match [13], which is the first necessary step towards precessional switching [12]. The most direct evidence for magnetization switching can be obtained from magnetooptical imaging using Kerr microscopy (KM) based on the rotation of the linear polarization of light upon reflection on a magnetized sample [19, 20]. However, the Kerr 
rotation angle is usually no more than a fraction of degree in GaMnAs [21]. Therefore, differential imaging and an exposure time of the order of $1 \mathrm{~s}$ have to be used [22]. It is well known that this rotation angle and thus the magnetic contrast can be enhanced by using multiple reflections in an additional anti-reflecting dielectric layer deposited onto the surface of metallic magnetic samples [19, 23, 24, 25]. However, this has not yet been evidenced with magnetic semiconductors in which the typical penetration depth of light can be of several hundreds of nm. Dielectric layers are also needed for SAW generation and detection. Since GaMnAs is weakly piezoelectric, interdigitated metallic transducers combs for SAW generation and detection need to be patterned onto a piezoelectric $\mathrm{ZnO}$ layer evaporated at high temperature $\left(150-250^{\circ} \mathrm{C}\right)$ on $\mathrm{GaMnAs}$ with a $\mathrm{SiO}_{2}$ buffer layer. However, this process might be detrimental since the magnetic properties of the GaMnAs layer highly depend on strain and heat treatment [22].

The point is then to take advantage of these dielectric layers to optimize the magnetic contrast while keeping the detrimental effect of this capping as low as possible.

In this paper, we investigate the wavelength and temperature dependence of the magnetooptical properties of a GaMnAs layer with and without capping by dielectric layers using polar magneto-optical Kerr effect (PMOKE).

\section{2 - Sample and experimental setup}

The sample is a $50 \mathrm{~nm}$ thick $\mathrm{Ga}_{1-\mathrm{x}} \mathrm{Mn}_{\mathrm{x}} \mathrm{As}(\mathrm{x}=0.07)$ layer grown on a $(\mathrm{Ga}, \mathrm{In}) \mathrm{As}$ relaxed buffer layer deposited on a semi-insulating (001) GaAs substrate in order to achieve perpendicular magnetic easy axis [22,26]. The (Ga,Mn)As layer was deposited at $250^{\circ} \mathrm{C}$. After the growth, the sample was thermally annealed at $250^{\circ} \mathrm{C}$ for 1 hour in order to improve its magnetic properties.

From SQUID magnetometry the Curie temperature was found to be $116 \mathrm{~K}$ and the active Mn concentration was about $3.3 \%$. One sample was kept as a reference (GaMnAs-ref). On one part of the second sample (named $\left.\mathrm{GaMnAs} / \mathrm{SiO}_{2} / \mathrm{ZnO}\right)$ a $\mathrm{SiO}_{2}(80 \mathrm{~nm}) / \mathrm{ZnO}(250 \mathrm{~nm})$ bilayer was grown by radio-frequency sputtering. The rest of the sample was masked during the growth of the dielectric layers. Care was taken to keep the sample holder at relatively low temperature $\left(150^{\circ} \mathrm{C}\right)$ in order not to further anneal the GaMnAs layer. The masked GaMnAs part (GaMnAs-h) is nevertheless checked. After optical investigation, the $\mathrm{ZnO}$ layer was removed with $\mathrm{HCl}$ leaving a $\mathrm{GaMnAs} / \mathrm{SiO}_{2}$ part. Eventually, $\mathrm{SiO}_{2}$ was further removed by hydrofluoric acid.

MOKE measurements were performed in the polar geometry with the light beam at quasinormal incidence on the sample surface. Monochromated light from a $150 \mathrm{~W}$ Xe lamp was polarized with a Glan-Thompson polarizer, chopped at $f_{0}=250 \mathrm{~Hz}$ by a mechanical chopper and phase-modulated at $f=50 \mathrm{kHz}$ by a photo-elastic modulator (PEM). The beam reflected off the sample passed through an analyzer, and was detected by a Si photodiode whose signal was fed into lock-in amplifiers. The signal at $f_{0}$ provided a measurement of the average light intensity at each wavelength. The Kerr rotation angle $\theta_{K}$ and the ellipticity $\eta_{K}$ were obtained from the signals at $2 f$ and $f$, respectively, following the procedure described in Ref. [27]. The sample was kept in a helium-flow cryostat, allowing the temperature $T$ to be varied from 10 to $300 \mathrm{~K}$. An external magnetic field was applied perpendicular to the sample surface and could be swept between $-20 \mathrm{mT}$ and $+20 \mathrm{mT}$. The KM images were obtained using a polarizing microscope in reflection geometry. The sample was illuminated at $\lambda=670 \mathrm{~nm}$, close to the maximum of the Kerr rotation angle. Hysteresis cycle can be obtained by averaging the signal over each image while scanning the magnetic field. Details can be found elsewhere [22]. 


\section{3- Results and discussion}

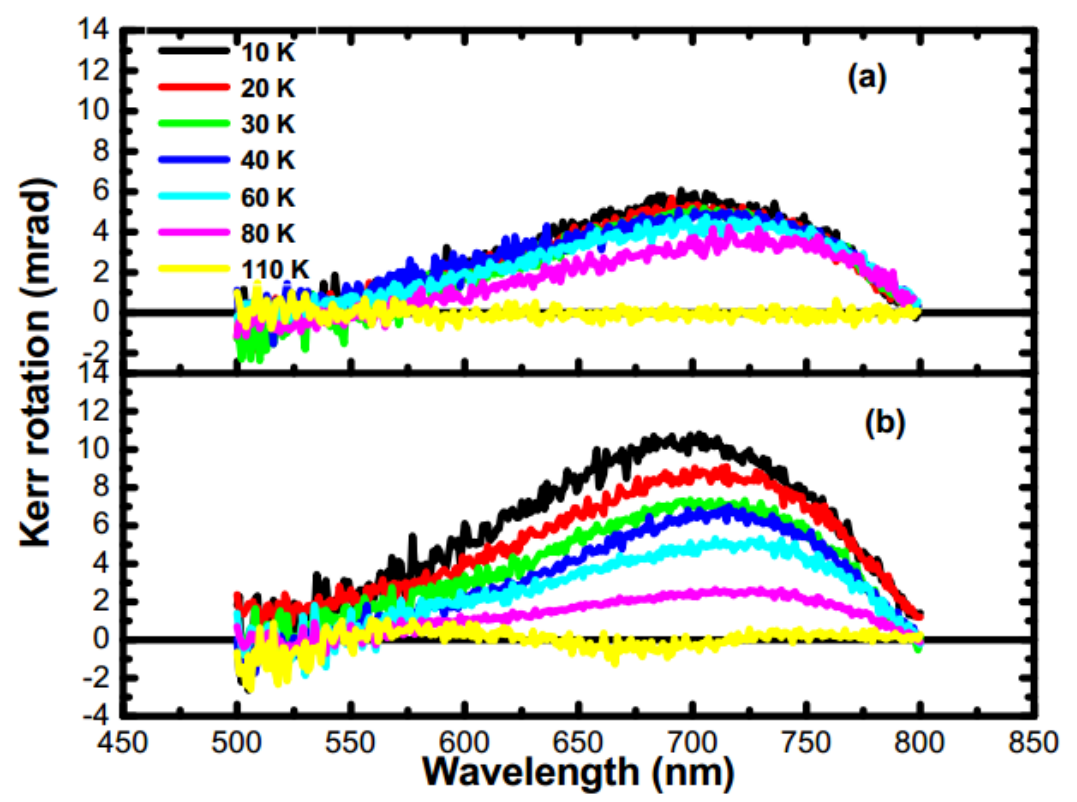

Fig.1. Kerr rotation spectra at different temperatures for GaMnAs (a) and $\mathrm{GaMnAs} / \mathrm{SiO}_{2}$ (b) layers.

First, samples GaMnAs-ref, GaMnAs-h, and $\mathrm{GaMnAs} / \mathrm{SiO}_{2} / \mathrm{ZnO}$ were studied. Hysteresis cycles were obtained from KM images at $T=30 \mathrm{~K}$. The first two samples show very similar hysteresis cycles. The coercive field was the same $(2.5 \mathrm{mT})$ however the saturation field was larger for GaMnAs-h indicating that the heating process has created defects acting as domain-wall pinning centers. The coercive field was slightly larger for $\mathrm{GaMnAs} / \mathrm{SiO}_{2} / \mathrm{ZnO}$ ( $3.3 \mathrm{mT}$ instead of $2.5 \mathrm{mT}$ ), so was the saturation field. In this sample, the magnetic contrast between the up and down magnetized states is similar to GaMnAs-h however the details of the magnetic domains pattern could hardly be distinguished. These results mean that in future SAW devices, the $\mathrm{ZnO}$ layer will have to be removed between the generating and detecting interdigitated combs in order to allow magneto-optical imaging of domains in the GaMnAs layer. We will therefore now concentrate on the magneto-optical properties of the different zones of the sample after $\mathrm{ZnO}$ removal, namely $\mathrm{GaMnAs} / \mathrm{SiO}_{2}$ and GaMnAs-h, hereafter simply called GaMnAs.

PMOKE spectra are shown in Fig. 1 for GaMnAs/SiO ${ }_{2}$ and GaMnAs. For both zones, the spectral dependence of the Kerr rotation $\theta_{K}$ is similar with a maximum around $704 \mathrm{~nm}$. It is clear that the Kerr rotation is enhanced by a factor 2 at $T=10 \mathrm{~K}$ for $\mathrm{GaMnAs} / \mathrm{SiO}_{2}$ with respect to GaMnAs. Such an effect, which will be discussed in more details in the next section, was already observed for other ferromagnetic materials (TbFe and GdTbFeCo with a-Si:0 [23], $\mathrm{NiFe}$ with $\mathrm{ZnS}$ [24]). $\mathrm{MgO}$ and $\mathrm{SiN}_{\mathrm{x}}$ capping layers on $\mathrm{CoFe}$ have also been investigated [25].

The amplitude of $\theta_{K}$ decreases with temperature. This point will addressed more quantitatively below.

Figure 2 shows the ellipticity $\eta_{K}$ versus wavelength for both samples at $T=10 \mathrm{~K}$. Both spectra have the same energy dip, at about $760 \mathrm{~nm}$. This dip is at lower energy relative to 
the $\theta_{K}$ peak. The ellipticity (in absolute value) is larger than the Kerr rotation (about 20 mrad at the dip position) however the enhancement with the $\mathrm{SiO}_{2}$ layer is not as high as for the Kerr rotation (21 mrad instead of $18 \mathrm{mrad}$ at the dip position) in agreement with calculations (see below).

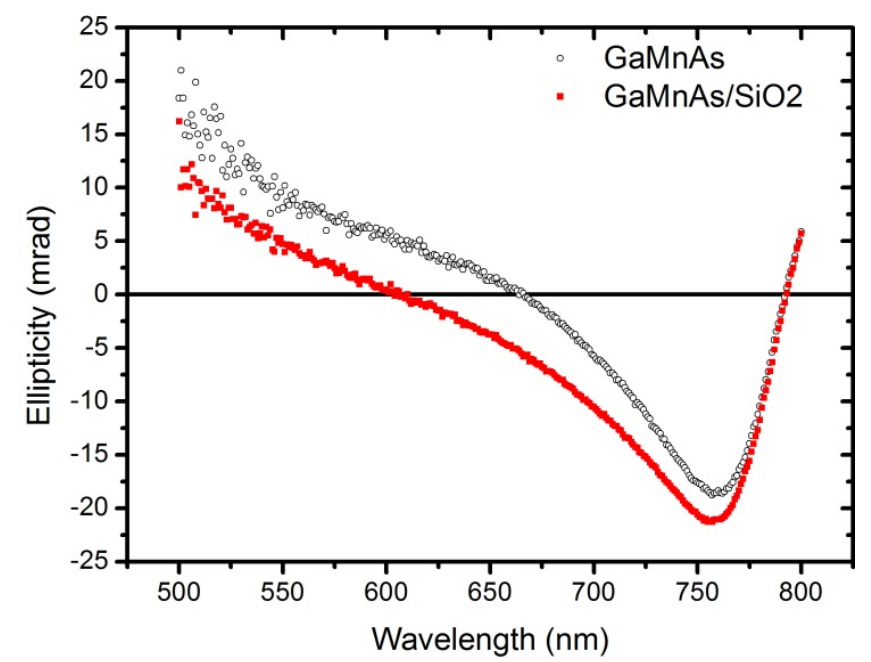

Fig.2. Ellipticity as a function of wavelength at $T=10 \mathrm{~K}$ for GaMnAs (black empty circles) and $\mathrm{GaMnAs} / \mathrm{SiO}_{2}$ (red squares).

In order to assess the usefulness of the $\mathrm{SiO}_{2}$ capping layer in the operating conditions for SAW devices we take Kerr images of the magnetic domains at different temperatures from $T=30 \mathrm{~K}$ to $T=90 \mathrm{~K}$ for $\mathrm{GaMnAs} / \mathrm{SiO}_{2}$ and GaMnAs layers (Fig.3).

In each image of Fig. 3, the GaMnAs and $\mathrm{GaMnAs}_{\mathrm{SiO}} / \mathrm{Si}_{2}$ zones are on the left and right parts of the image, respectively, as delimited by the dashed line. The sample edge is shown by the full line. These images are differential images. The reference is taken with the magnetization fully saturated in one direction. Therefore, the reversed domains appear on a uniform background. As typically observed for GaMnAs grown on a GaInAs pseudosubstrate [22], magnetization reversal proceeds with the nucleation and growth of dendritic like domains branching along the [110] and [-110] crystallographic directions. The contrast between the up and down states of magnetization is defined as

$C=2\left(I_{+}-I_{-}\right) /\left(I_{+}+I_{-}\right)$where $I_{+}$and $I_{-}$are the intensities of up and down magnetized domains, respectively. At $T=31 \mathrm{~K}$ domains appear as much brighter for $\mathrm{GaMnAs} / \mathrm{SiO}_{2}$. The magnetic contrast is enhanced from $5.6 \%$ to $11.3 \%$, i.e. by a factor of 2 , with the $\mathrm{SiO}_{2}$ capping layer on GaMnAs. However, the nucleation of domains and their expansion is more difficult, in agreement with the slightly larger coercive field and higher field needed to reach saturation. At higher temperature, domain nucleation occurs at the sample edge and propagation is not impeded. 


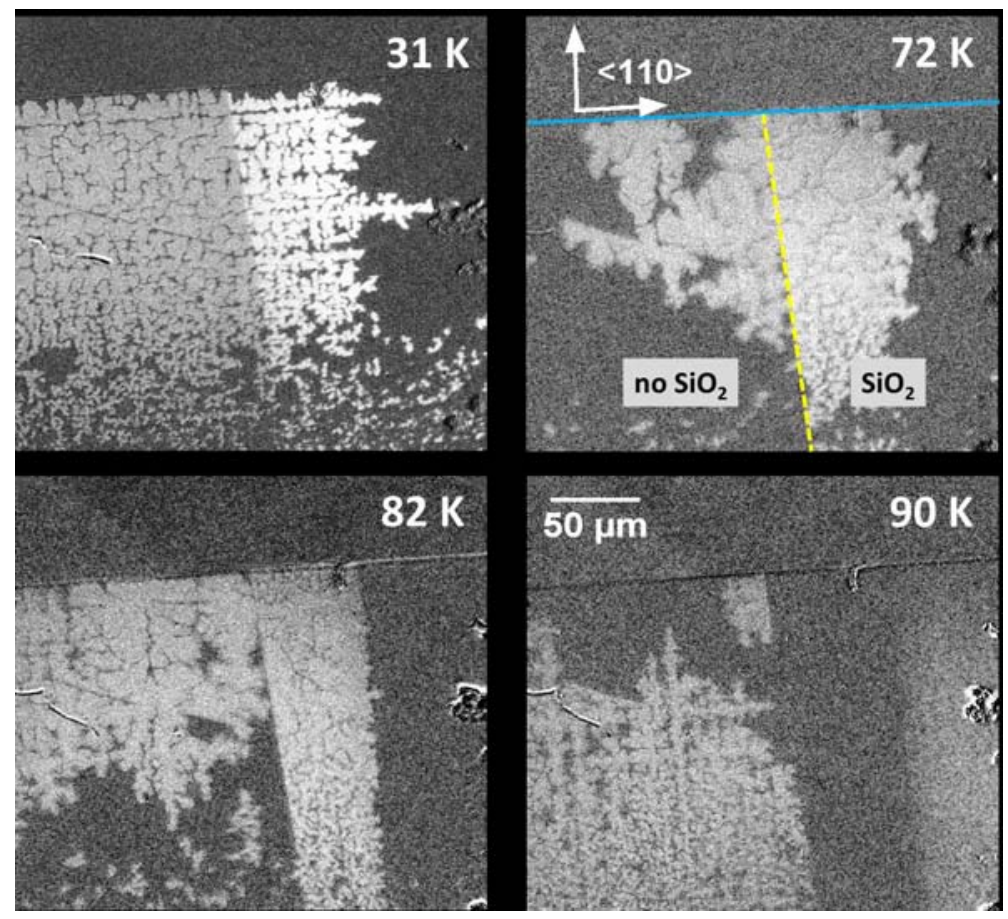

Fig.3.Snapshots of the magnetic domain structure observed by KM in a temperature range $30 \mathrm{~K}-90 \mathrm{~K}$, on the GaMnAs layer with and without $\mathrm{SiO}_{2}$ capping layer (right and left side of the dashed line, respectively). The sample edge is shown by a full line. Here, the magnetic contrast has been digitally enhanced with respect to the original images for the sake of clarity
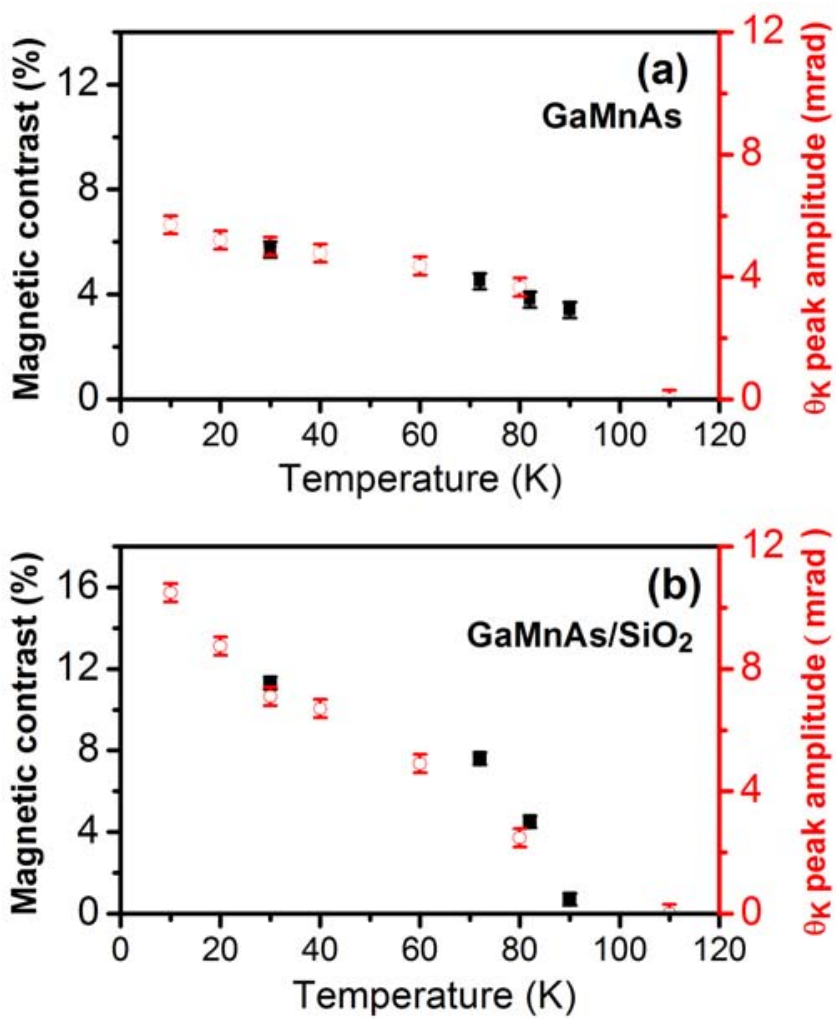
Fig.4. Dependence of the Kerr rotation peak amplitude (open circles) and the magnetic contrast of Kerr images (solid squares) on the temperature for GaMnAs (a) and $\mathrm{GaMnAs} / \mathrm{SiO}_{2}$ (b) layers.

Figure 4 shows the magnetic contrast of Kerr images together with the peak of the Kerr rotation angle obtained from PMOKE as a function of temperature for GaMnAs (Fig. 4 (a)) and $\mathrm{GaMnAs} / \mathrm{SiO}_{2}$ (Fig. 4 (b)). The magnetic contrast follows the same temperature dependence as the Kerr rotation. The proportionality factors between the contrast and the Kerr angle are not exactly identical for GaMnAs and $\mathrm{GaMnAs} / \mathrm{SiO}_{2}$ but close (1.2 and 1.5, respectively), although they should theoretically be equal in our experimental conditions. For $\mathrm{GaMnAs} / \mathrm{SiO}_{2}$, the contrast drops to zero at $T=90 \mathrm{~K}$, indicating a reduction of the Curie temperature from $116 \mathrm{~K}$ to $90 \mathrm{~K}$. A similar drop of $\mathrm{T}_{\mathrm{C}}$ has also been found from electrical measurements of the SAW attenuation and velocity change in a SAW-driven ferromagnetic resonance experiment in GaMnAsP [13]. After full removal of the $\mathrm{SiO}_{2}$ layer, $\mathrm{T}_{\mathrm{C}}$ remains at $93 \mathrm{~K}$ indicating that irreversible damages were produced as could also be found from X-rays measurements on a similar sample [13].

\section{4- Model}

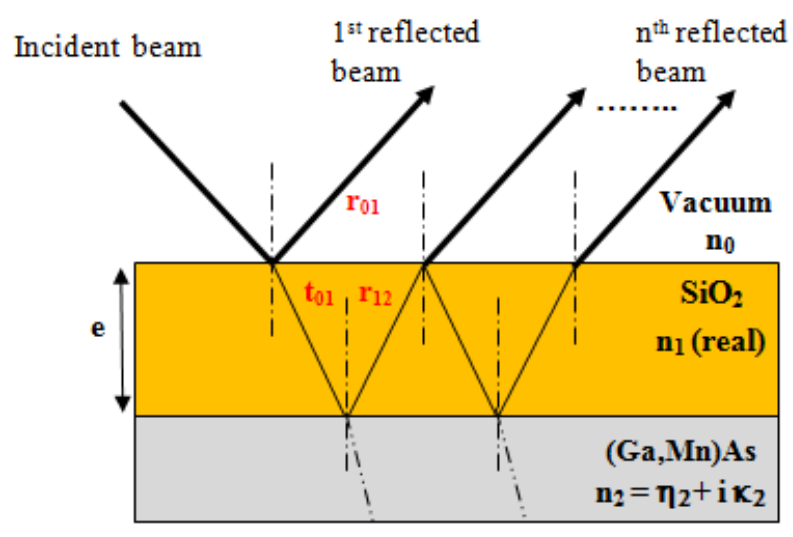

Fig.5.Schematic illustration of a model based on optical interferences in $(\mathrm{Ga}, \mathrm{Mn}) \mathrm{As} / \mathrm{SiO}_{2}$ structure. For the sake of clarity, the experimentally near-normal incidence light beam is shown here with a large incidence angle.

The experimental results will be discussed in the framework of an optical interference model as schematically depicted in Fig. 5. We assume that the GaMnAs layer has an infinite thickness, i.e. we do not take into account reflection at the substrate interface, which is weak owing to a very small difference of refractive indices [11]. The refractive index $n_{l}$ (real) of the silica layer is taken from [28]. The wavelength dependence of the real and imaginary parts of the GaMnAs refractive index $n_{2}=\eta_{2}+i \kappa_{2}$ are obtained from ellipsometry measurements on a similar sample at room temperature.

The amplitude reflection coefficient at normal incidence is written as:

$$
r=\frac{r_{01}+r_{12} \exp (\text { ite })}{1+r_{01} r_{12} \exp (\text { ite })}
$$


where $r_{01}=\left(1-n_{1}\right) /\left(1+n_{1}\right)$ and $r_{12}=\left(n_{1}-n_{2}\right) /\left(n_{1}-n_{2}\right)$ are the amplitude reflection coefficients at the vacuum $/ \mathrm{SiO}_{2}$ interface and at the $\mathrm{SiO}_{2} / \mathrm{GaMnAs}$ interface respectively. $e$ is the $\mathrm{SiO}_{2}$ thickness and $t$ is equal to $4 \pi n_{1} / \lambda$ with $\lambda$ the wavelength. It is expected that in the condition of anti-reflection, i.e. for a silica thickness close to a quarter of the wavelength in this material $\lambda /\left(4 n_{1}\right)$, multiple reflections at the silica/GaMnAs interface will enhance the Kerr rotation while decreasing the intensity of reflected light.

By minimizing the reflectance $R=r r^{*}$, one deduces the expression of the optimal thickness of the $\mathrm{SiO}_{2}$ layer:

$$
e_{o p t}=\frac{\lambda}{2 \pi n_{1}} \operatorname{ArcTan}\left(\frac{\operatorname{Re}\left(r_{12}\right)-\left|r_{12}\right|}{\operatorname{Im}\left(r_{12}\right)}\right) \approx \frac{\lambda}{2 \pi n_{1}} \operatorname{ArcTan}\left(\frac{\eta_{2}^{2}-n_{1}^{2}}{n_{1} \kappa_{2}}\right)
$$

where $\operatorname{Re}(\mathrm{Im})$ denotes the real (imaginary) part, respectively, and the approximate expression assumes a weak absorption in GaMnAs $\left(\kappa_{2}<<\left(\eta_{2}, n_{1}\right)\right)$. The optimal thickness shown in the inset of Fig. 6 , is found to be within a few percent $(4.5 \%$ to $1.8 \%)$ of the quarter wavelength $\lambda /\left(4 n_{1}\right)$ in the spectral range $\lambda=500 \mathrm{~nm}$ to $800 \mathrm{~nm}$.

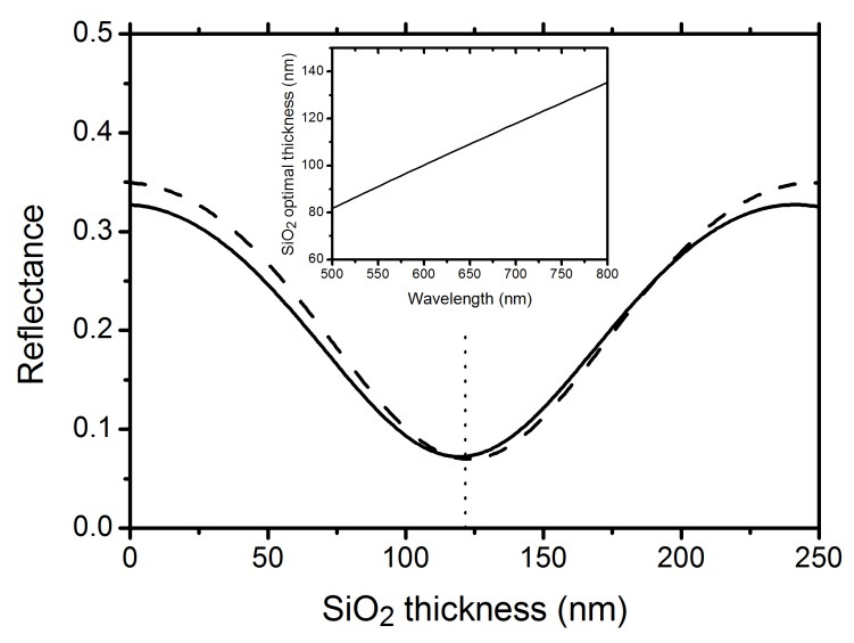

Fig. 6. Reflectance as a function of $\mathrm{SiO}_{2}$ thickness at the peak of Kerr rotation $(\lambda=704 \mathrm{~nm})$, for normal incidence (full line) and $20^{\circ}$ incidence (dashed line). The dotted vertical line shows the value of the $\mathrm{SiO}_{2}$ thickness equal to a quarter wavelength in the $\mathrm{SiO}_{2}$ layer $\mathrm{e}=\lambda /\left(4 \mathrm{n}_{1}\right)$. The inset shows the $\mathrm{SiO}_{2}$ thickness for minimum reflectance as a function of the wavelength (Eq. 2).

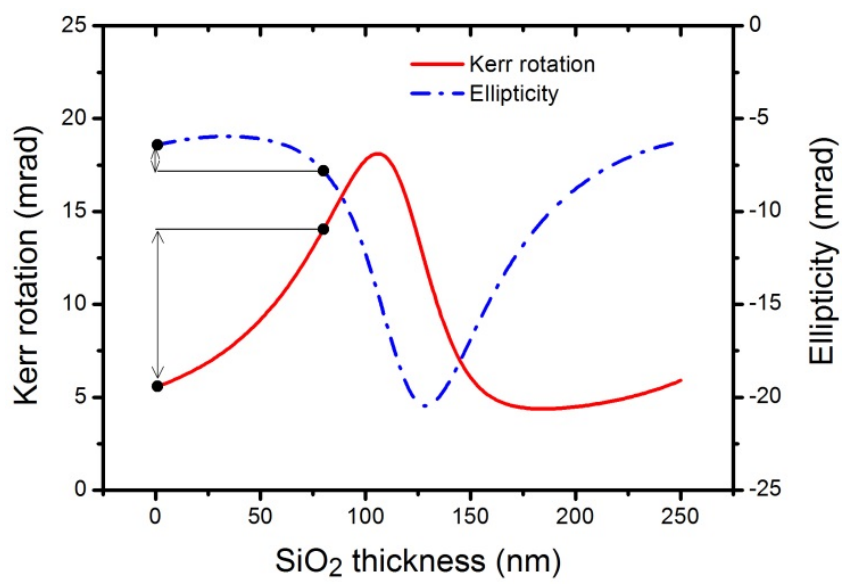


Fig. 7. Kerr rotation angle (solid line) and ellipticity (dashed-dotted line) at $704 \mathrm{~nm}$ (peak of the Kerr rotation) and $T=10 \mathrm{~K}$ as a function of the $\mathrm{SiO}_{2}$ thickness from Eq. 3. The arrows show the change of Kerr rotation and ellipticity from no $\mathrm{SiO}_{2}$ layer to a $80 \mathrm{~nm} \mathrm{SiO}_{2}$ layer.

The calculated reflectance is shown in Fig. 6 at $\lambda=704 \mathrm{~nm}$ (the peak of Kerr rotation) as a function of the silica thickness. The reflectance minimum is broad. The choice of the $\mathrm{SiO}_{2}$ thickness is therefore not critical. The thickness for minimum reflectance is $119 \mathrm{~nm}$. The reflectance has also been calculated for an angle of incidence of $20^{\circ}$ (the maximum incidence angle with the microscope objective used for KM). It is shown by the dashed line in Fig. 6. There is minor change with respect to normal incidence; which means that optimization of the silica thickness can be done without taking into account the numerical aperture of the objective.

The Kerr rotation and ellipticity are calculated as $\operatorname{Re}\left(\Theta_{K}\right)$ and $\operatorname{Im}\left(\Theta_{K}\right)$, respectively, where $\Theta_{K}$ reads:

$\Theta_{K}=-i \frac{Q\left(r_{01}^{2}-1\right)\left(1+r_{12}\right) t_{21} \exp (i t e)}{4\left(r_{01}+r_{12} \exp (i t e)\right)\left(1+r_{01} r_{12} \exp (i t e)\right)}$

where $Q=Q r+i Q i$ is the complex Kerr coefficient proportional to the saturation magnetization $M$. It is related to the off-diagonal element $\varepsilon_{x y}$ of the dielectric permittivity tensor by $\varepsilon_{x y}=-i \varepsilon_{2} Q m_{z}$, with $m_{z}$ the component of the unit magnetization vector along the normal to the sample surface and $\varepsilon_{2}=n_{2}^{2}$ [19]. The transmission coefficient $t_{21}$ is equal to $2 n_{2} /\left(n_{1}+n_{2}\right)$.

The $Q r$ and $Q i$ values are obtained from the experimental Kerr rotation and ellipticity at $T=10 \mathrm{~K}$. The dependence of the Kerr rotation and ellipticity on the $\mathrm{SiO}_{2}$ thickness at the peak of Kerr rotation $\lambda=704 \mathrm{~nm}$ is calculated and shown in Fig. 7. The arrows show the change of Kerr rotation and ellipticity from zero to $80 \mathrm{~nm}$ of silica. They are in good agreement with experimental results. One finds a change of the Kerr rotation from 5.6 $\mathrm{mrad}$ to $14 \mathrm{mrad}(10.5 \mathrm{mrad}$ experimentally) and of the ellipticity from $-6.4 \mathrm{mrad}$ to -7.8 $\mathrm{mrad}(-11 \mathrm{mrad}$ experimentally). In particular, the increase of the Kerr rotation is larger than the increase of Kerr ellipticity (in absolute value), as found experimentally. The precise knowledge of the GaMnAs refractive index at low temperature would certainly improve the agreement with experimental results. One can note that the peak of Kerr rotation is found for a silica thickness of $106 \mathrm{~nm}$, slightly smaller than the thickness for minimum reflectance $(119 \mathrm{~nm})$.

\section{5- Conclusion:}

In view of using dielectric $\mathrm{SiO}_{2}$ and $\mathrm{ZnO}$ layers as buffer layer and piezoelectric transducer, respectively, for surface acoustic wave generation and detection on a ferromagnetic semiconductor, we have grown a GaMnAs $/ \mathrm{SiO}_{2} / \mathrm{ZnO}$ sandwich and studied its magneto-optical properties. The aim was to assess the possible detrimental changes of the magnetic properties after the growth of overlayers on the GaMnAs ferromagnetic layer and to improve magneto-optical imaging for the study of SAW-assisted magnetization reversal. Although the magnetic properties of the tri-layer were not seriously altered, the $\mathrm{ZnO}$ layer did not allow good quality imaging of the magnetic domains. In future SAW devices, the $\mathrm{ZnO}$ layer will have to be removed in a window between the two sets of excitation and detection interdigitated combs. With a $\mathrm{SiO}_{2}$ layer of $80 \mathrm{~nm}$, the magnetic 
contrast could be multiplied by 2 . An optimized thickness of $106 \mathrm{~nm}$ should further increase the contrast. The temperature behavior of the magnetic contrast and the Kerr rotation angle has shown that the Curie temperature decreased from $116 \mathrm{~K}$ to $90 \mathrm{~K}$ with the $\mathrm{SiO}_{2}$ capping layer. This remains a problem but not too serious for SAW device operation. More generally this work can serve as a basis for optimization of magnetic contrast in magnetic semiconductors, not only for the investigation of SAW induced magnetization switching but also for the study of domain patterns and domain wall dynamics.

\section{Acknowledgement}

We are grateful to J. Y. Duquesne from Institut des Nanosciences de Paris for the growth of the dielectric layers on GaMnAs. This work has been supported by Region Ile-de-France (DIM Nano-K MURAS2012), by Agence Nationale de la Recherche (ANR 2010-BLANC0424-02 and ANR13-JS04-0001-01), and by the French RENATECH network. One of us, H. R., acknowledges financial support from the Ministry of Higher Education and Scientific Research of Tunisia. 
[1] P. Němec, V. Novák, N. Tesařová, E. Rozkotová, H. Reichlová, D. Butkovičová, F. Trojánek, K. Olejník, P. Malý, R. P. Campion, B. L. Gallagher, J. Sinova, and T. Jungwirth, Nature 4 ( 2013) 1422.

[2] T. Dietl, and H. Ohno, Review of Modern Physics 86 (2014) 187.

[3] A. W. Rushforth, E. De Ranieri, J. Zemen, J. Wunderlich, K. W. Edmonds, C. S. King, E. Ahmad, R. P. Campion, C. T. Foxon, B. L. Gallagher, K. Výborný, J.Kučera, and T. Jungwirth, Physical Review B 78 (2008) 085314.

[4] A. Lemaître, A. Miard, L. Travers, O. Mauguin, L. Largeau, C. Gourdon, V. Jeudy, M. Tran, and J.-M. George. Applied Physics Letters 93 (2008) 021123.

[5] K. Olejník, M. H. S. Owen, V. Novák, J. Mašek, A. C. Irvine, J. Wunderlich, and T. Jungwirth, Physical Review B 78 (2008) 054403.

[6] A. V. Kimel, G. V. Astakhov, G. M. Schott, A. Kirilyuk, D. R. Yakovlev, G. Karczewski, W. Ossau, G. Schmidt, L. W. Molenkamp, and T. Rasing, Physical Review Letters 92 (2004) 237203.

[7] G. V. Astakhov, A. V. Kimel, G. M. Schott, A. A. Tsvetkov, A. Kirilyuk, D. R. Yakovlev, G. Karczewski, W. Ossau, G. Schmidt, L. W. Molenkamp, and Th. Rasing, Applied Physical Letters 86 (2005) 152506.

[8] Y. Hashimoto and H. Munekata, Applied Physical Letters 93 (2008) 202506.

[9] P. Němec, E. Rozkotová, N. Tesařová, F. Trojánek, E. De Ranieri, K. Olejník, J. Zemen, V. Novák, M. Cukr, P. Malý, and T. Jungwirth, Nature Physics8 (2012) 411- 415.

[10] M.Bombeck, A. S. Salasyuk, B. A. Glavin, A. V. Scherbakov, C. Brüggemann, D. R. Yakovlev, V. F. Sapega, X. Liu, J. K. Furdyna, A. V. Akimov, and M. Bayer, Physical Review B 85 (2012) 195324.

[11] L. Thevenard, E. Peronne, C. Gourdon, C. Testelin, M. Cubukcu, E. Charron, S.

Vincent, A. Lemaître, and B. Perrin, Physical Review B 82 (2010) 104422.

[12] L.Thevenard, J.-Y. Duquesne, E. Peronne, H. J. von Bardeleben, H. Jaffres, S.

Ruttala, J-M. George, A. Lemaitre, and C. Gourdon, Physical Review. B 87 (2013)

144402.

[13] L. Thevenard, C. Gourdon, J. Y. Prieur, H. J. von Bardeleben, S. Vincent, L. Becerra, L. Largeau, and J.-Y. Duquesne, Physical Review B 90 (2014) 094401.

[14] J. Zemen, J. Kucera, K. Olejník, and T. Jungwirth, Physical Review B 80 (2009) 155203.

[15] M. Glunk, J. Daeubler, L. Dreher,S. Schwaiger, W. Schoch, R. Sauer, W. Limmer, A. Brandlmaier, S. T. B. Goennenwein, C. Bihler and M. S. Brandt, Physical Review B 79(2009) 195206.

[16] W. Li, P. Dhagat, and A. Jander, IEEE Trans. Magn48 (2012) 4100.

[17] W. Li, B. Buford, A. Jander, and P. Dhagat, Journal of Applied Physics 115 (2014) $17 \mathrm{E} 307$.

[18] S. Davis, J. A. Borchers, B. B. Maranville, and S. Adenwalla, Journal of Applied Physics $117(2015) 063904$.

[19] A. Hubert and R.Schäfer, Magnetic domains, Springer, Berlin (2009).

[20] A.K Zvezdin and V.A Kotov, Modern magneto-optics and magneto-optical materials, Studies in Condensed Matter Physics, Taylor \& Francis Group (New York), 1997.

[21] C. Sun, J. Kono, Y. -H. Cho, A. K. Wojcik, A. Belyanin, and H. Munekata, Physical Review B 83 (2011) 125206.

[22] A. Dourlat, V. Jeudy, C.Testelin, F. Bernardot, K. Khazen, C. Gourdon, L. Thevenard, L. Largeau, O. Mauguin, and A. Lemaître, Journal of Applied Physics102 (2007) 023913.

[23] K. Nakamura, T. Asaka, S. Asari, Y. Ota, and A. Itoh, IEEE Transactions on Magnetics MAG-21 (1985) 1654. 
[24] P. H. Lissberger, Journal of the Optical Society of America 51 (1961) 948, and references therein.

[25] K-M. Wu, J-F. Wang, K-C. Chen, J-C. Wu, and L. Horng. Journal of Magnetism and Magnetic Materials 310 (2007) e944-e946.

[26] L. Thevenard, L. Largeau, O. Mauguin, G. Patriarche, and A. Lemaître, Physical ReviewB 73 (2006) 195331.

[27] K. Sato, Japanese Journal of Applied Physics 20,(1981) 2403-2409.

[28] I. H. Malitson, Journal of the Optical Society of America 55, (1965) 1205. 


\section{Figure captions}

Fig.2.Kerr rotation spectra at different temperatures for GaMnAs (a) and $\mathrm{GaMnAs} / \mathrm{SiO}_{2}$ (b) layers.

Fig.2. Ellipticity as a function of wavelength at $T=10 \mathrm{~K}$ for GaMnAs (black empty circles) and $\mathrm{GaMnAs} / \mathrm{SiO}_{2}$ (red squares).

Fig.3. Snapshots of the magnetic domain structure observed by KM in a temperature range 30 $\mathrm{K}-90 \mathrm{~K}$, on the GaMnAs layer with and without $\mathrm{SiO}_{2}$ capping layer (right and left side of the dashed line, respectively). The sample edge is shown by a full line. Here, the magnetic contrast has been digitally enhanced with respect to the original images for the sake of clarity.

Fig.4. Dependence of the Kerr rotation peak amplitude (open circles) and the magnetic contrast of Kerr images (solid squares) on the temperature for GaMnAs (a) and $\mathrm{GaMnAs} / \mathrm{SiO}_{2}$ (b) layers.

Fig.5. Schematic illustration of a model based on optical interferences in $(\mathrm{Ga}, \mathrm{Mn}) \mathrm{As} / \mathrm{SiO}_{2}$ structure. For the sake of clarity, the experimentally near-normal incidence light beam is shown here with a large incidence angle.

Fig. 6. Reflectance as a function of $\mathrm{SiO}_{2}$ thickness at the peak of Kerr rotation $(\lambda=704 \mathrm{~nm})$, for normal incidence (full line) and $20^{\circ}$ incidence (dashed line). The dotted vertical line shows the value of the $\mathrm{SiO}_{2}$ thickness equal to a quarter wavelength in the $\mathrm{SiO}_{2}$ layer $\mathrm{e}=\lambda /\left(4 \mathrm{n}_{1}\right)$. The inset shows the $\mathrm{SiO}_{2}$ thickness for minimum reflectance as a function of the wavelength (Eq. 2).

Fig. 7. Kerr rotation angle (solid line) and ellipticity (dashed-dotted line) at $704 \mathrm{~nm}$ (peak of the Kerr rotation) and $T=10 \mathrm{~K}$ as a function of the $\mathrm{SiO}_{2}$ thickness from Eq. 3. The arrows show the change of Kerr rotation and ellipticity from no $\mathrm{SiO}_{2}$ layer to a $80 \mathrm{~nm} \mathrm{SiO}_{2}$ layer. 